\title{
Caracterizações de convexidades geométricas de grafos
}

\author{
Rafael Teixeira de Araújo ${ }^{1}$, Rudini Menezes Sampaio ${ }^{1}$ \\ ${ }^{1}$ Departamento de Computação - Universidade Federal do Ceará (UFC) \\ 60455760 - Fortaleza, CE - Brasil \\ rafaelteixeira@lia.ufc.br, rudini@lia.ufc.br
}

\begin{abstract}
A graph convexity is geometric if every convex set is the convex hull of its extreme vertices. Several papers have characterized classes of graphs for which a given convexity is geometric, such as the monophonic convexity in chordal graphs, the geodesic convexity in ptolemaic graphs [Farber and Jamison 1986] and the $m_{3}$-convexity in weak-biporized graphs [Dragan et al. 1999]. In this paper, we obtain characterizations for the convexities $P_{3}, P_{3}^{*}, P_{4}^{+}$and triangle-path. We also obtain results in the opposite direction: is there a graph convexity which is geometric exactly in a given graph class?
\end{abstract}

Resumo. Uma convexidade de grafos é geométrica se todo conjunto convexo é o fecho convexo de seus vértices extremos. Vários trabalhos têm caracterizado as classes de grafos para os quais uma dada convexidade é geométrica, como a convexidade monofônica em grafos cordais, a convexidade geodésica em grafos ptolemaicos [Farber and Jamison 1986] e a convexidade $m_{3}$ em grafos bipolarizados fracos [Dragan et al. 1999]. Nesse artigo, obtemos caracterizações para as convexidades $P_{3}, P_{3}^{*}, P_{4}^{+}$e triangle-path. Também obtemos resultados no sentido oposto: dada uma classe de grafos, existe alguma convexidade que só é geométrica nessa classe?

\section{Introdução}

Uma convexidade de grafos é um par ordenado $(G, \mathcal{C})$, onde $G$ é um grafo simples e $\mathcal{C}$ é uma coleção de subconjuntos de $V(G)$ (chamados conjuntos convexos) tal que $\emptyset$, $V(G) \in \mathcal{C}$ e a interseção de dois conjuntos convexos é convexo. Dado um grafo $G$ e uma coleção de caminhos $\mathcal{P}$ em $G$, a convexidade de caminhos $\mathcal{P}$ é tal que um conjunto $S \subseteq V(G)$ é convexo se e só se todo caminho em $\mathcal{P}$ com extremidades em $S$ está contido em $S$.

Entre as convexidades de caminho mais conhecidas, destacam-se a convexidade $P_{3}$ (caminhos com 3 vértices) [Campos et al. 2015], convexidade geodésica (caminhos mínimos) [Araújo et al. 2013], convexidade monofônica (caminhos induzidos) [Dourado et al. 2010], convexidade $m_{3}$ (caminhos induzidos com mais de 3 vértices) [Dragan et al. 1999] e convexidade triangle-path (T-caminhos: caminhos cujas cordas formam triângulos no caminho) [Changat and Mathew 1999].

Dada uma convexidade $(G, \mathcal{C})$, o fecho convexo $\mathcal{H}(S)$ de um conjunto $S \subseteq V(G)$ é o menor conjunto convexo que contém $S$. Dado um conjunto convexo $S \subseteq V(G)$, dizemos que $v \in S$ é um extremo de $S$ se $S \backslash\{v\}$ também é convexo. Uma convexidade $(G, \mathcal{C})$ 
é geométrica se satisfaz a propriedade de Minkowski-Krein-Milman: todo conjunto convexo é fecho convexo de seus extremos. Sabe-se que essa propriedade é equivalente a propriedade Antiexchange: para todo $S \subseteq V(G)$ e quaisquer vértices distintos $x, y \notin S$, temos que $x \in \mathcal{H}(S \cup\{y\})$ implica $y \notin \mathcal{H}(S \cup\{x\})$.

Uma questão bastante estudada é caracterizar a classe de grafos para os quais uma dada convexidade é geométrica.

\section{Resultados conhecidos}

O seguinte teorema obtém uma caracterização para a convexidade monofônica. Um grafo é cordal se não possui holes (ciclo induzido de tamanho maior que três).

Teorema 2.1 ([Farber and Jamison 1986]). A convexidade monofônica de um grafo Gé geométrica se e só se Gé cordal.

O seguinte teorema obtém uma caracterização para a convexidade geodésica. Um 3-fan é um grafo com 5 vértices tal que um vértice é universal e os demais induzem um $P_{4}$. Um grafo é ptolemaico se para quaisquer 4 vértices $a, b, x, y$ de uma mesma componente conexa:

$$
d(a, b) \cdot d(x, y) \leq d(a, x) \cdot d(b, y)+d(b, x) \cdot d(a, y) .
$$

Teorema 2.2 ([Farber and Jamison 1986]). Seja G um grafo. Então as seguintes propriedades são equivalentes: (a) a convexidade geodésica de G é geométrica; (b) Gé um grafo ptolemaico; (c) $G$ é cordal e não tem 3-fan induzido; (d) Gé cordal e todo caminho induzido é mínimo.

O teorema abaixo obtém uma caracterização para a convexidade $m_{3}$. Um grafo é bipolarizado-fraco se é livre de HHDA (livre de hole, house, dominó e o grafo A).

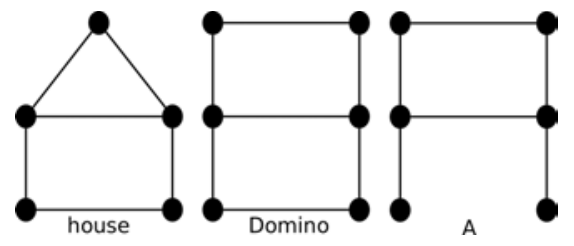

Figura 1. Grafos house, dominó, A

Teorema 2.3. [Dragan et al. 1999] A convexidade $m_{3}$ de um grafo $G$ é geométrica se e só se Gé um grafo bipolarizado fraco.

\section{Resultados novos}

Seguindo a sequência desses resultados, nós obtivemos em [Araújo and Sampaio 2013] alguns resultados novos: uma caracterização para as convexidades triangle-path, $P_{3}, P_{3}^{*}$ e $P_{4}^{+}$.

Dado um grafo $G$, um conjunto $S$ é $P_{3}^{*}$-convexo, se para todo $P_{3}$ induzido $a b c \mathrm{com}$ $a, c \in S$, então $b \in S$. Similarmente, definimos um conjunto $S$ como sendo $P_{4}^{+}$-convexo, se para todo $P_{4}$ induzido abcd com $a, c, d \in S$, então $b \in S$.

Teorema 3.1. Seja G um grafo. A convexidade triangle-path de Gé geométrica se e só se $G$ é uma floresta. A convexidade $P_{3}$ de $G$ é geométrica se e só se $G$ é uma floresta de estrelas. A convexidade $P_{3}^{*}$ de $G$ é geométrica se e só se $G$ é um cografo cordal. A convexidade $P_{4}^{+}$de Gé geométrica se e só se G é um cografo. 
Devido a restrição de espaço, faremos apenas as provas para as convexidades $P_{3} \mathrm{e}$ triangle-path.

Prova para Convexidade $P_{3}$. Suponha que a convexidade $P_{3}$ é geométrica em $G$. Se $G$ possui um $K_{3}\{x, y, z\}$, temos que $S=\{z\}$ é um conjunto convexo, $x \in \mathcal{H}(S \cup\{y\})$ e $y \in$ $\mathcal{H}(S \cup\{x\})$, o que contradiz a propriedade Antiexchange. Logo $G$ é livre de triângulos. Se $G$ possui um $C_{4}$ induzido $\{a, b, c, d\}$ com arestas $a b, b c, c d, d a$, temos que $S=\{c, d\}$ é um conjunto convexo pois $G$ é livre de triângulos e portanto não existe vértice adjacente a $c$ e $d$. Portanto, $a \in \mathcal{H}(S \cup\{b\})$ e $b \in \mathcal{H}(S \cup\{a\})$, o que contradiz a propriedade Antiexchange. Logo $G$ é livre de $C_{4}$. Se $G$ possui um $C_{5}$ induzido $\{a, b, c, d, e\}$ com arestas $a b, b c, c d, d e, e a$, temos que $S=\{c, d, e\}$ é um conjunto convexo (pois $G$ não possui $K_{3}$ nem $C_{4}$ induzido). Portanto, $a \in \mathcal{H}(S \cup\{b\})$ e $b \in \mathcal{H}(S \cup\{a\})$, o que contradiz a propriedade Antiexchange. Logo $G$ é livre de $C_{5}$ induzido.

Se $G$ possui um $P_{4}$ induzido wxyz, então $S=\{w, z\}$ é $P_{3}$-convexo. Isso porque se existisse um vértice $u$ adjacente a $w$ e $z$, teríamos que $u x$ e $u y$ não seriam arestas (pois $G$ é livre de triângulos) e portanto teríamos o $C_{5}$ induzido $\{w, x, y, z, u\}$, uma contradição. Portanto, $x \in \mathcal{H}(S \cup\{y\})$ e $y \in \mathcal{H}(S \cup\{x\})$, o que contradiz a propriedade Antiexchange. Logo $G$ é livre de $P_{4}$ induzido. Ou seja, $G$ é um cografo livre de $K_{3}$ e $C_{4}$.

Sabe-se que todo cografo conexo $G$ é a junção de dois cografos $G_{1}$ e $G_{2}$ (incluindo todas as arestas entre $G_{1}$ e $G_{2}$ ). Se $G_{1}$ possui uma aresta $a b$ então teríamos um $K_{3}\{a b z\}$ com qualquer vértice $z$ de $G_{2}$, uma contradição. Logo, $G_{1}$ e $G_{2}$ não possuem arestas. Se $G_{1}$ e $G_{2}$ possuem mais de 2 vértices cada, então teríamos um $C_{4}$ induzido $\left\{u_{1}, v_{1}, u_{2}, v_{2}\right\}$ com quaisquer vértices $u_{1}, v_{1} \in V\left(G_{1}\right)$ e $u_{2}, v_{2} \in V\left(G_{2}\right)$, uma contradição. Portanto, $G_{1}$ ou $G_{2}$ possui apenas um vértice. Portanto $G$ é uma estrela $K_{1, p}$. Se $G$ é desconexo, então $G$ é uma floresta de estrelas.

Se $G$ é uma estrela com pelo menos 3 vértices e centro $c$, então $c$ é o único vértice com grau maior que um e portanto todos os $P_{3}$ induzidos contém $c$. Isso implica que a propriedade Antiexchange é satisfeita e portanto a convexidade $P_{3}$ é geométrica.

Prova para convexidade triangle-path. Suponha que a convexidade triangle-path é geométrica em $G$. Se $G$ possui um $K_{3}$ induzido $\{x, y, z\}$, temos que $S=\{z\}$ é um conjunto convexo, $x \in \mathcal{H}(S \cup\{y\})$ (pois há o T-caminho $y x z$ entre $y$ e $z$ passando por $x$ com uma única corda $y z$ que forma um triângulo) e $y \in \mathcal{H}(S \cup\{x\}$ ) (pelo mesmo motivo anterior), o que contradiz a propriedade Antiexchange. Logo $G$ é livre de triângulos.

Se $G$ possui um ciclo induzido $v_{1}, v_{2}, \ldots, v_{k}$ para $k \geq 4$, temos que $S=\left\{v_{2}, v_{3}\right\}$ é um conjunto T-convexo, pois não existem T-caminhos entre $v_{2}$ e $v_{3}$ visto que $G$ é livre de triângulos. Note que $v_{1} \in \mathcal{H}\left(S \cup\left\{v_{4}\right\}\right)$ e $v_{4} \in \mathcal{H}\left(S \cup\left\{v_{1}\right\}\right)$, o que contradiz a propriedade Antiexchange. Logo $G$ é livre de ciclos induzidos. Como todo ciclo contém um ciclo induzido, temos que $G$ é livre de ciclos. Ou seja $G$ é uma floresta.

Por outro lado, suponha que $G$ é uma floresta. Logo $G$ não contém triângulos. Com isso, todo T-caminho de $G$ é um caminho induzido, e vice-versa. Ou seja, todo conjunto T-convexo de $G$ é monofonicamente convexo, e vice-versa. Isso quer dizer que a convexidade triangle-path e a convexidade monofônica são idênticas em $G$. Como $G$ é uma floresta, então $G$ é cordal, o que implica que a convexidade monofônica é geométrica em $G$ e portanto a convexidade triangle-path também é geométrica em $G$. 
Também obtivemos resultados no sentido inverso: dada uma classe de grafos, existe uma convexidade que é geométrica apenas nos grafos dessa classe?

Seja $H$ um grafo com pelo menos dois vértices. Dado um grafo $G$, dizemos que um conjunto $S \subseteq V(G)$ é $H$-free convexo se para todo $S^{\prime} \subseteq S$, $\left|S^{\prime}\right|=|V(H)|-1$ temos que, se $S^{\prime} \cup\{x\}$ induz um grafo $H$, então $x \in S$. Dada uma família $\mathcal{F}$ de grafos com pelo menos dois vértices, dizemos que um conjunto $S \subseteq V(G)$ é $\mathcal{F}$-free convexo se é $H$-free convexo para todo $H \in \mathcal{F}$.

Teorema 3.2. A convexidade $\mathcal{F}$-free é geométrica em $G$ se e só se $G$ é livre de $\mathcal{F}$.

Demonstração. Se $G$ não possui nenhum subgrafo induzido $H \in \mathcal{F}$, então por definição todo subconjunto $S \subseteq V(G)$ é $H$-free convexo. Portanto, a propriedade Antiexchange é sempre satisfeita e a convexidade é geométrica.

Suponha que $G$ possui um subgrafo induzido em $\mathcal{F}$. Seja $H \in \mathcal{F}$ um subgrafo induzido de $G$ com número mínimo de vértices. Sejam $S$ um conjunto de vértices de $G$ que induz $H, x, y$ dois vértices distintos de $S$ e $S^{\prime}=S \backslash\{x, y\}$. Note que $S^{\prime}$ é $\mathcal{F}$ free convexo, pela minimalidade de $H$. Note ainda que $y \in \mathcal{H}\left(S^{\prime} \cup\{x\}\right)$ e que $x \in$ $\mathcal{H}\left(S^{\prime} \cup\{y\}\right)$, o que contradiz a propriedade Antiexchange. Logo, a convexidade não é geométrica.

Corolário 3.1. Seja $\mathcal{F}=\left\{C_{2 k+1}: k=1,2,3, \ldots\right\}$ a família infinita dos ciclos ímpares. A convexidade $\mathcal{F}$-free é geométrica em $G$ se e só se $G$ é bipartido.

Corolário 3.2. Seja $\mathcal{F}=\left\{T K_{5}, T K_{3,3}\right\}$, onde $T$ representa topological minor, a família infinita dos grafos que podem ser obtidos a partir do $K_{5}$ ou do $K_{3,3}$ através de subdivisão de arestas. A convexidade $\mathcal{F}$-free é geométrica em $G$ se e só se $G$ é planar.

\section{Referências}

Araújo, R. T., Sampaio, R., and Szwarcfiter, J. (2013). The convexity of induced paths of order three. Electronic Notes in Discrete Mathematics, 44(0):109 - 114.

Araújo, R. T. and Sampaio, R. (2013). Convexidades de caminhos e convexidades geométricas. Dissertação de Mestrado (MDCC-UFC), Fortaleza, Brazil.

Campos, V., Sampaio, R. M., Silva, A., and Szwarcfiter, J. L. (2015). Graphs with few $\mathrm{P}_{4}$ 's under the convexity of paths of order three. Discrete Applied Mathematics, 192:28 - 39. 11th Cologne/Twente Workshop on Graphs and Combinatorial Optimization (CTW 2012).

Changat, M. and Mathew, J. (1999). On triangle path convexity in graphs. Discrete Mathematics, 206(1-3):91 - 95.

Dourado, M. C., Protti, F., and Szwarcfiter, J. L. (2010). Complexity results related to monophonic convexity. Discrete Applied Mathematics, 158(12):1268 - 1274.

Dragan, F. F., Nicolai, F., and Brandstädt, A. (1999). Convexity and HHD-free graphs. SIAM Journal on Discrete Mathematics, 12(1):119-135 (electronic).

Farber, M. and Jamison, R. (1986). Convexity in graphs and hypergraphs. SIAM Journal on Algebraic Discrete Methods, 7(3):433-444. 